\title{
Identificación y selección de una herramienta tecnológica para la evaluación del desempeño académico en la Escuela de Armas Combinadas del Ejército
}

\author{
Jairo Augusto Vásquez Clavijo \\ Braymer Sinay Velásquez Carrillo \\ Andrés David Villa Molano \\ Alexander Román Yela Garzón \\ Escuela de Armas Combinadas del Ejército
}

\section{RESUMEN}

En este artículo académico se expone el proceso de investigación que se realizó para identificar la herramienta tecnológica adecuada que permita evaluar los cursos de ascenso de la Escuela de Armas Combinadas del Ejército (ESACE). Con este fin se revisaron los procesos evaluativos de la ESACE, las herramientas Blackboard y la plataforma CEDOC 360, empleadas actualmente en los cursos de ley en la Escuela. Asimismo, se realizó una investigación documental, se aplicaron encuestas a una muestra de los capitanes en curso de ley y se hicieron entrevistas semiestructuradas a personal de la oficina de tecnología de la ESACE. El análisis de la información obtenida permite concluir que la herramienta de evaluación adecuada para estos cursos es la plataforma Ultra, la cual está vinculada a las Blackboard que forman parte de la plataforma CEDOC 360 del Ejército Nacional.

\section{PALABRAS CLAVE}

Blackboard; educación; CEDOC 360; ESACE; evaluación de la educación; evaluación del estudiante; prueba de conocimientos.

\section{CITACIÓN}

Vásquez Clavijo, J. A., Velásquez Carrillo, B. S., Villa Molano, A. D., \& Yela Garzón, A. R. (2020). Identificación y selección de una herramienta tecnológica para la evaluación del desempeño académico en la Escuela de Armas Combinadas del Ejército7. Brújula, Semilleros de Investigación, 8 (15), 7-29. https://doi.org/10.21830/23460628.43 


\section{Introducción}

La inclusión de herramientas tecnológicas como parte de los procesos educativos era una idea que, desde un punto de vista persona, podía representar la evolución de la educación e incluso la materialización de la globalización. De la misma forma, otros autores la plantean como la innovación aplicada a la educación, por ejemplo, Héctor Emilio Dávila Rosero (2017), quien afirma que la innovación educativa colombiana está enfocada en el mejoramiento de la calidad de la educación, y menciona que la innovación conlleva nuevas formas de aprendizaje que se basan en la "familiaridad y apropiación del uso de tecnologías” (p. 11).

Asimismo, la vinculación de las Tecnologías de la Información y la Comunicación (TIC) ha generado un impacto notable en la sociedad mundial y colombiana, el mismo que a los ojos de autores como Hernández (2017) responde a las necesidades de una nueva sociedad, la que él llama "la sociedad del conocimiento y la tecnología” (p. 327). Esta se explica a partir de la premisa que incita al ser humano a comprender la dinámica de su entorno y a responder a la necesidad de buscar conocimientos útiles para adaptarse a los cambios que le impone. En otras palabras, la obligación de crear y obtener la información con la que el ser humano pueda ser competitivo dentro de una sociedad en la que el conocimiento y la información son el pilar de la competitividad, la proyección y el desarrollo.

No obstante, el uso de las tecnologías dentro de los ambientes académicos no es algo que atañe exclusivamente a los centros de educación básica o superior, pues estas herramientas también son parte de los procesos educativos de las Fuerzas Militares de Colombia, que propenden por la formación de profesionales competentes en un entorno cambiante y que se transforma en nuevos retos que evalúan continuamente las capacidades de los integrantes del Ejército Nacional, la Fuerza Aérea y la Armada Nacional.

En el caso del Ejército Nacional, un ejemplo de la importancia que tienen las competencias digitales en la institución y en los procesos de formación es la vinculación de aulas virtuales y herramientas de evaluación que nutren los métodos de aprendizaje y enseñanza para los alumnos que se encuentran en los cursos de ley. Según Londoño (2015), los estudiantes pueden aumentar sus capacidades para el aprendizaje a través del uso de las TIC y al mismo tiempo para evaluar en qué nivel de aprendizaje se encuentran, si en realidad están preparados para enfrentar los retos intelectuales y prácticos que la sociedad contemporánea impone (p. 5).

Por esta razón, se considera que el empleo de herramientas tecnológicas en la evaluación del desempeño académico tiene un papel fundamental en la actualidad, puesto que coadyuva al militar en la adquisición de habilidades para desplegar iniciativas que le permiten estar a la vanguardia en esta sociedad orientada al conocimiento tecnológico, además de que proporciona al Ejército Nacional herramientas que mejorar la calidad de vida de sus hombres y de quienes están en medio de un proceso de educación superior.

Del mismo modo, se encuentra que el uso de la tecnología en las escuelas de formación y capacitación del Ejército Nacional se ha convertido en un elemento fundamental que integra los procesos educativos desde la planificación y evaluación de los saberes académicos hasta el seguimiento del alumno militar. Asimismo, aporta una serie de beneficios que ayudan al estudiante a mejorar la eficiencia y la productividad en cada uno de los saberes.

Teniendo en cuenta lo anterior, se reconoce que la tecnología aporta beneficios en la educación, los cuales facilitan la generación de 
conocimientos a partir de la interacción entre los estudiantes. En este sentido, la gestión del aula ya no es solo misión del docente, sino que se apoya en el uso de las nuevas plataformas tecnológicas, en la optimización del tiempo durante la planificación académica a través de software que ayuda a mejorar la distribución de los horarios de estudio y a implementar estrategias flexibles. Asimismo, permite la mejora continua a través del uso de programas que optimizan el manejo de las mallas curriculares, planes de estudios, programas y syllabus de los diferentes cursos, con lo cual facilita además el proceso de acreditación y asegura la coherencia con los objetivos declarados en el perfil del egresado.

Adicionalmente, se considera que la tecnología genera mayor comunicación y gestión de los docentes con la implementación de software que abarque un seguimiento no solo a las acciones de los docentes en relación con las clases, sino también a otros aspectos como la capacitación continua o la investigación. Además, permite una gran reducción en los costos, ya que no es necesario el material gráfico impreso, pues todo se realiza a través de una plataforma o programa.

También se debe tener en cuenta que el desarrollo de las TIC ha generado una dinámica mundial en la que el uso de redes sociales y de dispositivos inteligentes se convirtió en un fenómeno con gran influencia en la sociedad. Todas las personas esperan conectarse desde diferentes partes del mundo a través de las redes, claro está, con el acceso a internet que poco a poco fue llegando a las empresas y a las casas. Así, el impacto de la tecnología y de sus avances ha sido cada vez mayor, de manera que las TIC no solamente se han integrado a los procesos de aprendizaje y al ámbito académico, sino que además han ampliado las oportunidades para grupos que por varios motivos no podían acce- der a un centro educativo, pero sí tenían a su alcance herramientas tecnológicas que facilitan la educación virtual (Nieto, 2012). Esto coadyuvó para que fuera realidad un escenario que se había pensado improbable: la tecnología, el internet, las redes sociales y los portales web se convierten en la herramienta adecuada para que las personas que no pueden asistir a un aula de clase puedan obtener un título en la distancia y complementen las posibilidades que ya ofrecía la educación abierta y a distancia. Cabe recordar que esta última surgió como consecuencia de la demanda insatisfecha de un grupo de personas que por diferentes situaciones debían buscar una alternativa para estudiar (García, s. f).

El surgimiento de una propuesta diferente para la educación abierta y a distancia también se convierte en un aliado para quienes estudian de manera presencial, ya que pueden complementar su proceso a través de las ofertas de aprendizaje basadas en TIC y otras herramientas tecnológicas con las que pueden construir el conocimiento a su propio ritmo y, también, evaluar su proceso haciendo uso de diferentes aplicaciones y software.

Las Fuerzas Armadas de Colombia, específicamente el Ejército Nacional, no pueden ser ajeno a este tipo de avances. Por el contrario, debe tener en cuenta que las TIC son parte de los procesos de formación y capacitación de oficiales, suboficiales y soldados, quienes deben demostrar capacidades que evidencien su competitividad y preparación para enfrentar los retos y desafíos de la profesión militar. Por esa razón, la inclusión de herramientas tecnológicas en los procesos de evaluación del desempeño académico se convierte en una ayuda para identificar problemáticas, falencias y puntos por mejorar, los cuales aportan conocimiento y experiencia para formar profesionales militares competentes y competitivos. 
No obstante, los aplicativos tecnológicos implementados para evaluar el desempeño académico del personal en formación constituyen una ayuda, no siempre tiene un buen rendimiento o a veces es difícil navegar en ellos porque los estudiantes no entienden del todo su diseño, lo cual dificulta el acceso a las evaluaciones y hace que los resultados no sean los esperados.

Con base en estas reflexiones se determinó que la problemática principal de este trabajo es la inclusión de la tecnología dentro de los procesos de evaluación del desempeño académico del personal que se forma en las diferentes escuelas de las Fuerzas Armadas colombianas. Específicamente, la pregunta problema de la investigación es la siguiente: ¿Cuál es la herramienta tecnológica apropiada para evaluar el desempeño académico de los capitanes en la Escuela de Armas Combinadas (ESACE) del Ejército Nacional?

En este sentido, el objetivo general es identificar una herramienta tecnológica apropiada para evaluar el desempeño académico de los capitanes en la ESACE. Este objetivo se apoya en los siguientes objetivos específicos: (1) analizar los procesos de evaluación de desempeño implementados actualmente en los cursos de ley de la ESACE; (2) definir las herramientas tecnológicas implementadas en los cursos de ascenso que se realizan en la ESACE y, por último, (3) proponer una herramienta tecnológica para evaluar el desempeño académico aplicable en los cursos de ascenso en la ESACE.

\section{Justificación}

Las necesidades cambiantes de la población que habita el territorio nacional, sumadas a los requerimientos en materia de seguridad y a los desafíos propios de la misión que constitucionalmente le fue encomendada a los integrantes del Ejército Nacional de Colombia, evidencian la obligación de formar profesionales competentes, que tengan conocimiento en materia militar y en diferentes ámbitos académicos que atañen a su labor. Por eso es necesario implementar herramientas que, de la mano con la tecnología, coadyuven en la formación de profesionales capacitados para que contribuyan desde sus conocimientos empíricos, militares y académicos en la construcción de una sociedad mejor y más segura.

Sin embargo, cabe señalar que implementar herramientas tecnológicas en los procesos de formación a veces se torna complejo por situaciones como la presentación de la plataforma y la facilidad para navegar en ella. Así mismo, por la dificultad para conectarse cuando las condiciones o la capacidad de internet no es suficiente, lo cual dificulta la continuidad de los cursos, cumplir con las fechas de entrega o cargar documentos.

En este sentido, es importante que el oficial desarrolle habilidades en el manejo de las herramientas tecnológicas utilizadas para evaluar su desempeño académico en cualquiera de los cursos de ley que pueda tomar. Así, en cumplimiento del numeral 3.2. "Experticia Militar" de la Doctrina Damasco, cuando habla de la formación de líderes multiplicadores de conocimiento (Ejército Nacional de Colombia, 2017), el personal en formación debe ser evaluado constantemente y corregir las falencias que la evaluación le señale, no solo para que ejerza bien este liderazgo en la transmisión de conocimiento, sino fundamentalmente para que el conocimiento que transmita sea correcto.

Dado que esta evaluación no tendrá el efecto esperado si no se efectúa a través de la herramienta apropiada, el propósito de esta investigación es fortalecer la evaluación del desempeño académico de los oficiales en curso de ascenso 
de capitán a mayor en la ESACE. Para esto es necesario identificar las brechas existentes dentro de los cursos y los aspectos que deben mejorar en los procesos de formación, así como verificar las debilidades y fortalezas de cada alumno con el fin de establecer los medios apropiados para fortalecer estos puntos débiles.

\section{Estado del arte}

Es innegable que en los últimos años el uso de herramientas tecnológicas ha sido una estrategia que fortalece la educación, sobre todo cuando se trata de la educación militar y de implementar evaluaciones que permiten establecer el nivel académico y de aprendizaje que han logrado los alumnos durante o después de una fase de instrucción en los cursos de ascenso del Ejército Nacional.

En la revisión de estudios previos sobre el tema se encuentra que varios autores han dirigido su atención al estudio de las TIC en los procesos de evaluación de estudiantes en diferentes niveles educativos, así como también en la construcción del conocimiento, la naturaleza cambiante de los ambientes de aprendizaje, entre otras. Algunos de los antecedentes que se relacionan con este tema son artículos, ensayos y otros textos, que, si bien no abordan específicamente la evaluación académica de militares en cursos de ley, sí estudian innovaciones con TIC en los procesos de evaluación del desempeño académico de estudiantes en diferentes niveles educativos.

Al respecto, Rodríguez (2005) analiza la influencia que ejercen las TIC en los procesos de evaluación de estudiantes universitarios de Europa. En primer lugar, considera que esto es resultado de las características de los entornos de aprendizaje y de los cambios metodológicos en la educación superior europea. Posteriormente, plantea la posibilidad de hacer evalua- ciones más eficientes que simplifiquen el trabajo de docentes y estudiantes a través de las TIC (Rodríguez, 2005). Finalmente, la autora concluye que es necesario seguir usando herramientas como internet para evaluar a los alumnos y hacer retroalimentaciones más completas por medio de este instrumento.

Por su parte, el trabajo de Vence (s. f.), realizado en el Departamento del Atlántico a través del programa Todos a Aprender, se centra en estudiar las posibilidades que tienen los profesores para mejorar los procesos de enseñanza y evaluación por medio de la evaluación interactiva. De acuerdo la autora, esta herramienta no solo instruye a los niños y adultos en el manejo de las tecnologías, sino que además proporciona guías para que los docentes aprovechen correctamente las TIC en los procesos de enseñanza y para que el estudiante asuma con mayor facilidad los temas y presente mejores resultados en las evaluaciones (Vence, s. f.).

Desde una perspectiva comunicacional, Ríos y Krenn (2015) plantean la necesidad de implementar estrategias que preparen a los estudiantes para afrontar los cambios que les impone el entorno. De acuerdo con las autoras, esta es la principal motivación para que los docentes vinculen las TIC a sus procesos de enseñanza, ya que permiten formar profesionales capaces de transformar positivamente su contexto y de construir sus aprendizajes valiéndose de los recursos que la tecnología y la innovación les proporciona en el mundo contemporáneo (Ríos \& Krenn, 2015).

Lafuente (2003) analiza los procesos de evaluación implementados en la educación virtual de dos centros universitarios para diagnosticar el uso que hacen de las herramientas tecnológicas en educación virtual y semipresencial. A este trabajo se suma el de Toro (2016), que hace un recorrido por la evolución de la educación 
y señala la manera como se diversificó con la inclusión de las TIC. Esta investigación se basó en el análisis de quinientas experiencias educativas con métodos innovadores realizadas entre 2001 y 2014 que modificaron positivamente los procesos de enseñanza y aprendizaje. Asimismo, los resultados de este trabajo sentaron las bases para el diseño de posteriores proyectos relacionados con la educación superior innovadora y el aprovechamiento de las herramientas tecnológicas dentro de los procesos de la enseñanza tecnológica (Toro, 2016).

Entre tanto, Christen y Ortiz (s. f.) presentan los resultados de un estudio que sirve como punto de partida para analizar las tendencias actuales en el uso de las TIC para evaluar el aprendizaje y verificar las habilidades y competencias de los alumnos, así como para mejorar los formatos de evaluación del aprendizaje. A la vez, Canales (2007) sistematiza buenas prácticas apoyadas en TIC en escenarios educativos que buscan la innovación y concluye que en los diferentes centros educativos que estudió hay un ambiente propicio para usar las TIC en el fomento de buenas prácticas y verificar del aprendizaje del conocimiento por medio de las evaluaciones (Canales, 2007).

De la misma forma, Guzmán (2008) analiza la aplicabilidad y pertinencia de las TIC en los procesos de enseñanza y aprendizaje en la Universidad de Querétaro para formular estrategias que permitan a la institución integrarlas a los distintos procesos educativos, incluyendo el de la evaluación. Por su parte, López (2009) analiza los cambios que la educación superior ha tenido en Europa como consecuencia del desarrollo de nuevas estrategias metodológicas que fomentan la construcción autónoma del conocimiento y el progreso de herramientas tecnológicas dentro de este proceso. El autor concluye que estas herramientas web cumplen funciones específicas enfocadas en la evaluación de los contenidos (López, 2009).

Finalmente, la Organización de las Naciones Unidas para la Educación, la Ciencia y la Cultura (Unesco, 2013) propone fortalecer el aprendizaje por medio de las TIC y su uso en procesos de aprendizaje. Específicamente, los investigadores de la Unesco se refieren a dos focos de atención priorizada, uno de los cuales es la evaluación de los aprendizajes por medio de las TIC, que ofrece herramientas con métodos innovadores que facilitan el seguimiento y la revisión de los aprendizajes de los estudiantes y, por ende, la medición del desempeño de los docentes que participan en su formación (Unesco, 2013).

\section{Fundamentación teórica, jurídica y conceptual}

El estudio de la evaluación académica encuentra cierta relación con teorías que pueden explicar la necesidad de implementar acciones tecnológicas en el proceso de evaluación académica del personal que se encuentra en curso de ascenso en la Escuela de Armas Combinadas del Ejército Nacional. También, con el postulado que demuestra la pertinencia de la idea de combinar las TIC con la evaluación de los conocimientos de los oficiales del Ejército Nacional, quienes necesitan prepararse para los retos que el entorno cambiante les presenta. En este sentido, las teorías aplicables a este estudio son la teoría del modelo constructivista, la teoría de la tecnología educativa, la teoría práctica sobre la evaluación y, por último, la teoría sobre la evaluación educativa.

E primer lugar, el constructivismo hace referencia a la idea de construir el conocimiento y con este el aprendizaje humano. Este tipo de aprendizaje responde a una teoría basada en la autonomía y se diferencia de otras porque 
proporciona al alumno los elementos suficientes para construir conocimiento por sí mismos (Hernández, 2008). Desde esta perspectiva, los estudiantes son sujetos activos dentro de la dinámica del aprendizaje y la enseñanza, de modo que contribuyen con la transformación del conocimiento, que no solo se obtiene a través de la transmisión docente-alumno, sino que también se consigue en la experiencia. Esta, al ser conjugada con la información previa, los datos y la información obtenida desde el aprendizaje autónomo, genera modelos mentales que se complementan con los datos que el ser humano continúa adquiriendo y le dan las bases al alumno para asimilar mejor su entorno y los cambios que se producen en él (figura 1) (Hernández, 2008, p. 27).
Esta teoría constructivista, aplicada al objeto de estudio de este artículo, evidencia a través de la evaluación con herramientas tecnológicas la capacidad que tiene cada uno de los oficiales en curso de ascenso para construir el conocimiento a partir de la experiencia que ha obtenido en el desempeño de su labor militar, los datos que le ha proporcionado los docentes y la información que ha obtenido de la lectura autónoma de documentos necesarios para elaborar textos escritos e investigaciones en la construcción del conocimiento que, a la postre, les será útil para la toma de decisiones y otras funciones propias del comandante.

Asimismo, la aplicación de la teoría constructivista en la evaluación del desempeño académico de los oficiales en cursos de ley nutre

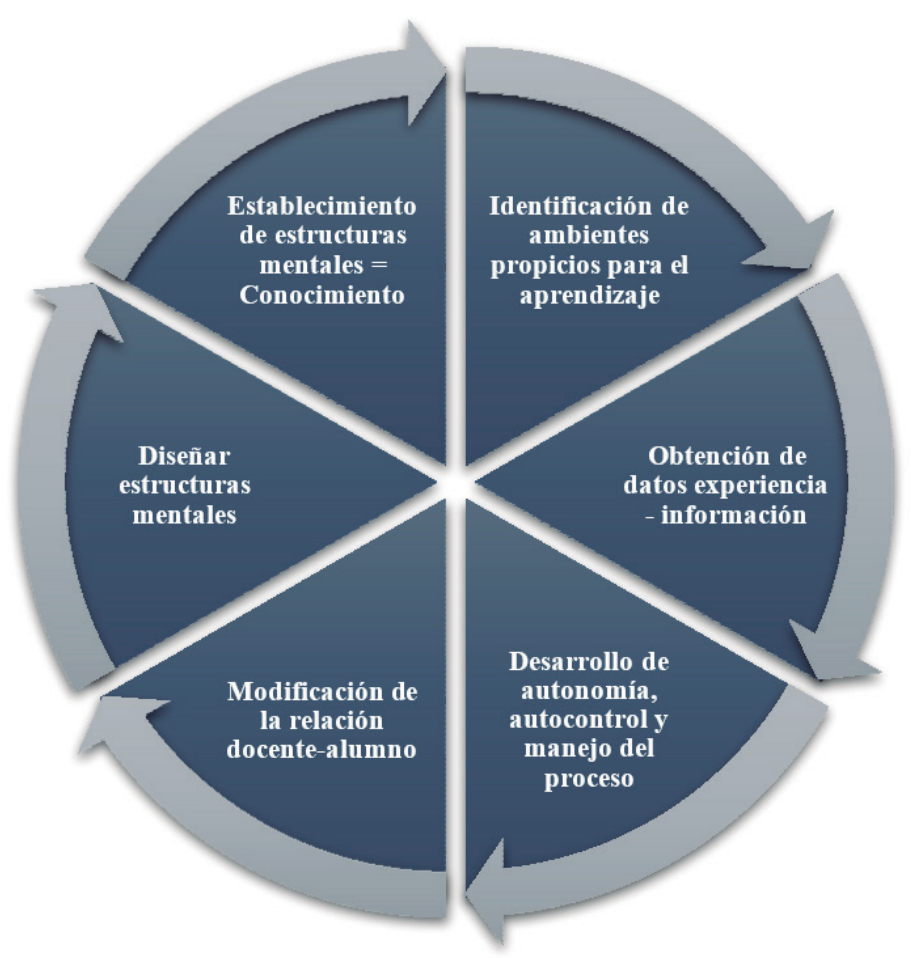

Figura 1. Proceso de la teoría constructivista Fuente: Original de los autores. 
los resultados obtenidos en la medición del conocimiento que han desarrollado los alumnos y que los convierte en profesionales competentes y propositivos. Este proceso tiene lugar en una institución que utiliza los datos conseguidos por medio de estas evaluaciones para reestructurar la enseñanza y el aprendizaje en el Ejército Nacional y la ESACE en pro de mejorar la calidad de la enseñanza que proporciona a sus alumnos. Así, las Fuerzas Armadas construyen conocimiento que se traduce en lecciones aprendidas y buenas prácticas con las que robustecen los programas aplicados en los cursos y establecen los parámetros de formación de los oficiales del Ejército Nacional.

Por su parte, si bien la teoría de la tecnología educativa no es reconocida, sí es pertinente al objeto de estudio de este documento por cuanto se relaciona con la construcción autónoma y considera que el espacio virtual es una herramienta propicia para cumplir los fines educativos contemporáneos. Por consiguiente, esta teoría encuentra en las plataformas virtuales, también llamadas "entornos virtuales de aprendizaje”, un espacio para que el ser humano pueda potenciar prácticas que mejoren los esquemas de aprendizaje y procesos pedagógicos por medio de aplicaciones e instrumentos en línea, los cuales evalúan el nivel de cumplimiento de las metas del aprendizaje y reconocen el tipo de conocimiento que ha creado el estudiante (García et ál., 2015).

Igual que sucede con la teoría constructivista, la teoría de la tecnología educativa es pertinente en este estudio porque enfoca su atención en el uso que se hace de los espacios de aprendizaje virtual para convertirlos en herramientas propicias para evaluar los conocimientos que los alumnos u oficiales han logrado construir. Asimismo, el resultado de estas herramientas nutren la planificación y los resultados de la evaluación de los saberes académicos con los que se miden la eficiencia y la productividad en cada uno de los saberes, así como la capacidad que desarrolló el alumno para construir los modelos de estructuras mentales.

Otro de los postulados que explica la intención de este estudio y la importancia de mejorar las condiciones de la evaluación académica en la ESACE proviene de la teoría práctica sobre la evaluación. Esta teoría se basa en la experiencia pedagógica de aproximadamente diez años de un profesor, que a través de un estudio de caso espera demostrar que la experiencia se convierte en parte de la teoría. En otras palabras, es la materialización de las expresiones verbales de un docente (García, 1988).

De acuerdo con el autor, esta es una teoría permanente que estudia la evaluación de la enseñanza y la forma como la evaluación de la teoría teórico-práctica es intrínseca a la experiencia obtenida por los docentes en el ejercicio de su labor. Con base en este estudio, García (1988) pretende explicar cómo la racionalidad encuentra una relación con la práctica, la cual, a la postre, se convierte en conocimiento racional que parte de la experiencia y que permite identificar las fortalezas y debilidades en el proceso de enseñanza; el mismo que posteriormente se convierte en la raíz del conocimiento que es evaluado por el docente (p. 234).

En lo que respecta a esta investigación, está teoría se puede abordar desde dos perspectivas diferentes: la primera de ellas, mencionada anteriormente, permite evaluar la enseñanza desde la visión del docente y la manera como este enseña los contenidos a los alumnos en curso de ascenso para concientizarlos acerca del vínculo que existe entre la teoría y la práctica. También sirve para revisar los medios que se implementan para evaluar el conocimiento de los alumnos que toman estos cursos. La segunda perspec- 
tiva de esta teoría tiene en cuenta la visión del alumno, mediante la cual puede explicar la conexión que se forja entre la teoría y la tecnología, que, en última instancia, se convertiría en la práctica en un mundo tecnificado y que necesita de la tecnología para innovar y mejorar la labor de los oficiales del Ejército Nacional.

Por último, se debe enfatizar que uno de los principales objetivos de esta investigación es la evaluación de los conocimientos que ha obtenido el grupo de capitanes en curso de ascenso. Por esta razón se tiene como punto de referencia el trabajo de Santos (1999), quien plantea que la evaluación forma parte de lo que él llama "cinco más cinco”, que no es más que la suma de las cinco funciones de la evaluación, las cinco sugerencias y las cinco dificultades (figura 2).

Santos (1999) fundamentó su teoría en el estudio de la patología de la evaluación educativa, metodología que le permitió identificar las limitaciones, condiciones y otras manipulaciones de las que es objeto la evaluación. También expresa las consecuencias que trae consigo el planteamiento negativo de esta herramienta, las cuales son expresadas por medio de la supervisión de la práctica docente y de las metodologías implementadas para que los alumnos aprendan y tengan buenos resultados dentro de los procesos que evalúan el desempeño académico de cada estudiante.

Esta teoría, aplicada al objeto de estudio de esta investigación, no solo demuestra la importancia de la evaluación en el proceso de aprendizaje de los oficiales, sino que también evidencia que para identificar desde las TIC la herramienta evaluativa apropiada es imprescindible tener claras las funciones de la evaluación, las sugerencias y las dificultades del proceso y de los recursos que se emplean en la actualidad. Precisamente, con base en esta información se diseñó y se podría perfeccionar el instrumento evaluativo más apropiado para los alumnos que propone esta investigación.

Ahora bien, este estudio no solo se basa en estas teorías. En Colombia y en el Ejército Nacional las leyes tienen un papel importante a la hora de determinar las acciones o iniciativas pertinentes para mejorar la calidad educativa. Sin embargo, es importante señalar que las normas que atañen al uso de las TIC en ambientes académicos en Colombia es un asunto diferente, de manera que la legislación se jerarquizó

- Poner en tela de juicio la forma de evaluar

- Investigar sobre los procesos de evaluación

- Desarrollo del diálogo profesional

- La participación de los alumnos en su evaluación

- La relación con los padres sobre la evaluación

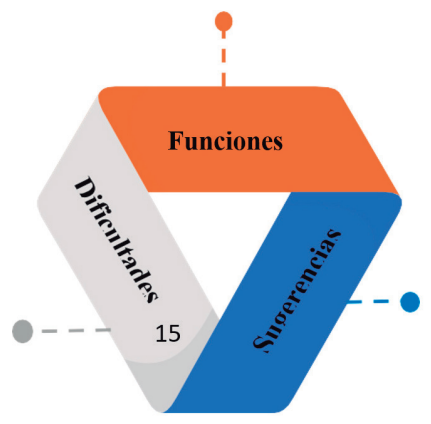

- La escasez de tiempo

- La falta de motivación

- Individualismo profesional

- La rutinización institucional

Figura 2. Teoría de la evaluación educativa (cinco más cinco). Como diagnóstico, selección, jerarquización, comunicación y formación.

Fuente: Original de los autores con base en Santos (1999). 
teniendo en cuenta la Pirámide de Kelsen, a partir de la cual y se establecieron las más sobresalientes (figura 3).

Estas normas se aplican de la siguiente manera:

- Constitución Política 1991: en el Artículo 67 se establece que la educación es un derecho fundamental de todos los colombianos. También señala que el Estado, la sociedad y la familia son los responsables de la educación de los niños, niñas y jóvenes colombianos. Al mismo tiempo, establece que es debe ser un propósito de la sociedad y del Estado mejorar y fortalecer la educación tecnológica en el territorio nacional.

- Ley 1341 de 2009. "Por la cual se definen principios y conceptos sobre la sociedad de la información y la organización de las Tecnologías de la Información y las Comunicaciones (TIC), se crea la Agencia Nacional de Espectro y se dictan otras Disposiciones” (República de Colombia, 2009): en el artículo 2, numeral 7 , se decreta el derecho a la comunicación, la información y la educación y los servicios básicos de la TIC que garantiza el acceso a las tecnologías de la información y la comunicación en la recepción de la educación y el conocimiento.

- Ley 115 de 1994. "Por la cual se expide la Ley General de Educación” (República de Colombia, 1994): es la ley que rige la educación en el país y establece los parámetros para ejercer la educación en el territorio nacional. El artículo 4 decreta que el Estado deberá proveer los medios para llevar a cabo procesos de promoción docente, provisión de recursos y métodos educativos, así como la inspección y evaluación de los procesos educativos.

- Ley 30 de 1992. "Por medio de la cual se organiza el servicio público de la Educación Superior” (República de Colombia, 1992): en el artículo 12 establece que "los programas de maestría, doctorado y posdoctorado tienen a la investigación como fundamento y

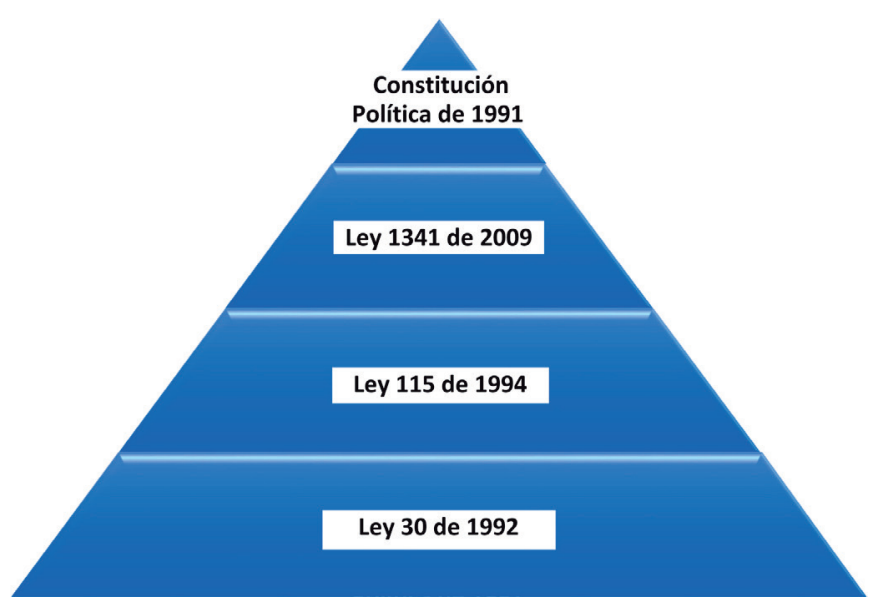

Figura 3. Marco jurídico según Pirámide de Kelsen. Fuente: Original de los autores. 
ámbito necesarios de su actividad. Las maestrías buscan ampliar y desarrollar los conocimientos para la solución de problemas disciplinarios, interdisciplinarios o profesionales y dotar a la persona de los instrumentos básicos que la habilitan como investigador en un área específica de las ciencias o de las tecnologías o que le permitan profundizar teórica y conceptualmente en un campo de la filosofía, de las humanidades y de las artes”. (República de Colombia, 1992)

Finalmente, la aplicación de las TIC en los procesos educativos de los oficiales del Ejército Nacional debe ser explicada desde una perspectiva conceptual, razón por la cual a continuación se definen los conceptos clave:

- Competitividad: surge con la globalización, son las ventajas que un actor posee sobre otro y que se materializan con las ventajas comparativas; estas ventajas se convierten en habilidades para no solo superar al rival; sino también para generar habilidades que mejoren la calidad de la gestión de cada persona, empresa, organización, etc. (Rojas y Sepúlveda, 1999). Aplicado al tema de estudio, la competitividad se entiende como las habilidades que debe desarrollar cada oficial para enfrentar y superar las expectativas en el cumplimiento de la misión institucional. Asimismo, se considera que estas habilidades se logran en la construcción del conocimiento y se perciben en la evaluación del desempeño académico.

- Conocimiento tecnológico: comúnmente se conoce como los saberes relacionados con la manipulación de las he- rramientas tecnológicas. Dentro de sus principales características están: es codificable, es transmisible, es observable, es dependiente y es verificable (Caro, s. f.). En el caso de este trabajo, el conocimiento tecnológico hace referencia a la información que cada alumno posee sobre el manejo de las herramientas proporcionadas por la ESACE para el aprendizaje y evaluación de sus conocimientos militares y académicos.

- Evaluación del desempeño académico: la evaluación del desempeño académico no es más que un proceso que se implementa en la finalización de cada curso de ley y en el que se miden los conocimientos obtenidos, las brechas, las fallas y las fortalezas de cada estudiante.

- Herramientas tecnológicas: son los instrumentos implementados para utilizar de manera eficiente de la tecnología (Schorborgh, 2009). En esta investigación, las herramientas tecnológicas son las plataformas virtuales, el software, las aplicaciones y demás espacios virtuales en donde los alumnos de los cursos de ley pueden desarrollar actividades $\mathrm{y}$ evaluaciones para medir su conocimiento, conocer sus debilidades y fortalezas.

- Tecnologías de la Información y la Comunicación: conocidas también como TIC, son definidas por la Unesco (2017) como las herramientas que desempeñan un papel fundamental "en el acceso universal a la educación, la igualdad en la instrucción, la enseñanza y el aprendizaje de calidad, la formación de docentes, y la gestión, dirección y administración más eficientes del sistema educativo" (Unesco, 2017, pág. 18). Desde el enfoque de esta investigación se considera 
que las TIC son las herramientas tecnológicas con las que se lleva a cabo los procesos de evaluación y con las que se reconocen las fortalezas y debilidades de los alumnos militares.

\section{Evaluación de desempeño en la Escuela de Armas Combinadas del Ejército Nacional}

De acuerdo con el artículo 137 de la Ley 3092 de 1992, las escuelas de formación de las Fuerzas Militares y de Policía continúan adscritas a las entidades respectivas (República de Colombia, 1992):

Artículo 137. La Escuela Superior de Administración Pública (Esap), el Instituto Tecnológico de Electrónica y Comunicaciones (Itec), el Instituto Caro y Cuervo, la Universidad Militar Nueva Granada, las Escuelas de Formación de las Fuerzas Militares y de la Policía Nacional que adelanten programas de Educación Superior y el Servicio Nacional de Aprendizaje (SENA), continuarán adscritas a las entidades respectivas. Funcionarán de acuerdo con su naturaleza jurídica y su régimen académico lo ajustarán conforme lo dispuesto en la presente ley. (p. 25)

Por consiguiente, la Ley de Educación Superior concede cierto tipo de autonomía a las escuelas de formación de las Fuerzas Militares, de manera que el Ministerio de Defensa Nacional cuenta con la potestad para definir el Plan Estratégico del Sistema Educativo de las Fuerzas Armadas (Pese) (Ministerio de Defensa Nacional, 2008). En ese documento el Ministerio de Defensa Nacional establece que la evaluación de los conocimientos es parte de los componentes que aseguran la calidad educativa de las fuerzas. Así mismo, señala que la búsqueda de la calidad en la formación de militares y policías colombianos debe estar alineada con los avances sociales, económicos y tecnológicos de la Nación y la región (Ministerio de Defensa
Nacional, 2008, p. 17). En otras palabras, es menester que los programas educativos ofrecidos en cada una de las escuelas de formación estén a la vanguardia de la educación educativa y, también, de las necesidades de la sociedad. Se entiende entonces que las necesidades de la sociedad determinan las competencias que se deben fomentar y desarrollar en los profesionales de las Fuerzas Armadas de Colombia.

Ahora bien, dado que la ESACE forma parte de las escuelas de formación del Ejército Nacional, se colige que los principios, los valores y demás parámetros de gestión que establece el Plan Estratégico (Ministerio de Defensa Nacional, 2008) se deben aplicar en los cursos de ley que imparte este centro educativo. Por lo tanto, es deber de docentes y demás encargados del área académica la formulación de evaluaciones de carácter académico con las cuales sea posible medir los conocimientos obtenidos por el personal en curso.

De la misma manera, el Plan Estratégico establece que uno de los parámetros para regular la educación profesional militar y policial es la aplicación correcta de procesos, subprocesos y actividades que permitan a los centros educativos como la ESACE garantizar la calidad educativa y satisfacer las necesidades de cada fuerza para que enseñen y evalúen los conocimientos que harán más competentes a los oficiales del Ejército Nacional (Ministerio de Defensa Nacional, 2008, p. 39).

En este sentido, la ESACE goza de las garantías y obligaciones que este sistema proporciona, como facilitar "el diseño de estructuras y estrategias para potenciar el aprendizaje autónomo del militar o policía, mediante la creación de múltiples ambientes de aprendizaje y de interactividades con soportes tecnológicos diversos” (Ministerio de Defensa Nacional, 2008, p. 17). Por esta razón, la ESACE deberá contar con 
métodos evaluativos para impulsar el aprendizaje autónomo que estén a la vanguardia de la evolución educativa y se adapten a la era tecnológica. Sin embargo, dado que se trata de un proceso que toma tiempo y exige recursos, con los cuales la Escuela no siempre cuenta, en el actual Curso Intermedio (Ascenso de Capitán a Mayor) las evaluaciones se presentan todavía con el método antiguo: papel y lápiz.

\section{Herramientas tecnológicas implementadas en los Cursos de Ascenso que se realizan en la Escuela de Armas Combinadas del Ejército Nacional}

Con base en la sistematización de la experiencia personal, de la revisión documental y de la aplicación de instrumentos de investigación como entrevistas y encuestas, se encuentra que las principales herramientas tecnológicas implementadas actualmente en la ESACE son Blackboard y la plataforma CEDOC 360.

\section{Blackboard}

A primera vista y partiendo de la experiencia personal, la Blackboard se relaciona con dos términos: investigación y encuestas. No obstante, la revisión documental sobre esta herramienta tecnológica y la revisión de sus utilidades muestran que su funcionalidad va más allá de conectar con repositorios o bases de datos académicas. Desde un punto de vista técnico, la Blackboard se define como una plataforma educativa a través de la cual los estudiantes, valiéndose de una conexión a internet y una cuenta institucional, tienen acceso a un curso determinado que se lleva a cabo de forma virtual. Dentro de las principales funciones de esta herramienta se encuentra: el contenido del curso o material de ayuda, un link para subir las tareas, espacios de comunicación con los docentes y compañeros (mensajería directa, chat), foros académicos, biblioteca virtual y evaluaciones en línea (Vicerrectoría de Asuntos Académicos Universidad del Turabo, s. f).

En contraste con esta información, se encuentra que el curso de ascenso de capitán a mayor emplea la Blackboard solamente como fuente de información, a pesar de que sus funciones podrían mejorar el ambiente de aprendizaje de los alumnos y se podría implementar como medio de evaluación, de tal forma que los alumnos desarrollen todo tipo de revisiones para conocer las falencias que tienen en los procesos educativos e identificar fortalezas y oportunidades.

\section{Plataforma CEDOC 360}

La plataforma CEDOC 360 es un proyecto que inició en el año 2017 como respuesta a las necesidades educativas y de transformación institucional para formar a los Héroes Multimisión. Se trata de una plataforma que mejora los procesos educativos dentro del Ejército Nacional, al tiempo que protege los datos de quienes se encuentran registrados. Además, el manejo de la comunicación a través de los correos institucionales proporciona espacios para hacer seguimiento y control a los proyectos investigativos por medio del módulo de investigación, entre otros (Centro de Educación Militar [Cemil], 2017).

Una de las principales características de esta herramienta es que integra otras plataformas como Blackboard, Renata, Sofiaplus (SENA) y Bibliotecas Virtuales. Asimismo, vincula instrumentos como Google Drive y cuenta con las siguientes funciones: registro y control, correos académicos; oferta académica; gestión de investigación; horarios y docentes; notas; evaluación y estadística, y bibliotecas (Cemil, 2017). 
Si bien es cierto que CEDOC 360 contribuye con las políticas ecológicas a través de la disminución del uso del papel y reduce costos para la fuerza en materia de alquiler de plataformas web y que, además, en el 2018 se agregaron otras funciones como una sección de peticiones, quejas, reclamos y sugerencias (PQRS), objetos virtuales de aprendizaje, generación de diplomas, entre otros (Cemil, 2017), la plataforma aún necesita otras acciones que pueden complementar los procesos educativos en la ESACE de acuerdo con la búsqueda de calidad en los procesos educativos de la Escuela y del Ejército Nacional.

\section{Métodos}

\section{Enfoque de la investigación}

Tomando como punto de referencia el trabajo de Hernández, Fernández y Baptista (2014), esta investigación identifica desde un enfoque cualitativo una herramienta TIC apropiada para evaluar del desempeño académico de los capitanes del Ejército Nacional en curso de ascenso. De acuerdo con los autores, en este enfoque la recolección y análisis de los datos puede reorientar la investigación a nuevos interrogantes durante el proceso de interpretación. Es por esto que se aplicó un instrumento para sistematizar y analizar los datos para desarrollar la pregunta problema de este proyecto, así para identificar una herramienta TIC y describirla, pero no medir su impacto en los procesos de evaluación de desempeño.

Asimismo, es importante tener en cuenta que esta investigación sigue un proceso inductivo que busca explorar las distintas posibilidades que tienen las TIC en la evaluación del desempeño académico de los capitanes en curso de ascenso a mayor en la ESACE. Para esto, inicia con la exploración del entorno, continúa con la descripción de las herramientas TIC vinculadas con la evaluación de los conocimientos y el aprendizaje de los alumnos y, por último, genera una propuesta en la que se sugiere una herramienta apropiada para realizar esta evaluación con mayor facilidad y alienación con las políticas institucionales.

\section{Tipo de investigación, recolección de datos y fuentes de investigación \\ De acuerdo con Hernández, Fernández y} Baptista (2014), la recolección de datos se realiza mediante la revisión documental en textos como libros, artículos académicos, estudios previos y revisión de documentos institucionales que contienen información relacionada con el tema. Además, señalan que esta revisión se complementa con instrumentos de investigación para recoger datos a través de encuestas y entrevistas semiestructuradas, mediante las cuales se podrá determinar la posición que tienen los estudiantes frente a las herramientas TIC implementadas, sus expectativas sobre la vinculación de las herramientas tecnológicas dentro de su proceso de formación para el siguiente grado y lo que esperan de estas herramientas.

Del mismo modo, las entrevistas semiestructuradas permiten conocer la posición de quienes trabajan en la oficina de tecnología y están relacionados con la evaluación de contenidos a través de herramientas web como Blackboard respecto a la posibilidad de encontrar una herramienta propicia para evaluar el desempeño académico de los capitanes que se encuentran en el curso de ley en la ESACE. Es importante mencionar que los datos que se obtienen a través de cada instrumento son analizados para confirmar la viabilidad y pertinencia de la investigación, así como para sentar las bases que sirven como referencia para formular la propuesta final y la medición del alcance del proyecto. 
Por último, cabe agregar que esta investigación recurre a fuentes de investigación primarias, definidas por Hernández, Fernández y Baptista (2014) como aquellas que "proporcionan datos de primera mano, pues se trata de documentos que incluyen los resultados de los estudios correspondientes” (p. 61). Específicamente, en esta investigación estas fuentes corresponden a estudios previos, crónicas y noticias periodísticas, las encuestas, las entrevistas semiestructuradas y la normatividad que pudiera influir dentro del análisis del fenómeno.

\section{Muestra poblacional}

Como se ha mencionado a lo largo del documento, la población objetivo son los estudiantes de la ESACE, de manera que este es el universo de la investigación. No obstante, fue necesario focalizar la investigación en el grupo de capitanes que se encuentran en curso de ley en esta cohorte, lo cual lleva a definir como muestra poblacional al grupo de capitanes en curso de ascenso en la ESACE. Teniendo en cuenta lo anterior, las encuestas se aplicaron a una muestra poblacional de 146 de los 234 capitanes de la ESACE, con un nivel de confianza del 95\% y un margen de error del 5\%.

\section{Resultados}

Para desarrollar esta investigación se aplicaron dos instrumentos de investigación que complementan la revisión documental para cumplir los objetivos específicos n. ${ }^{\circ} 1 \mathrm{y}$ n. ${ }^{\circ}$ 3: el primero es una encuesta aplicada al curso de ascenso de capitán a mayor de la ESACE (anexo 1) y el segundo es una entrevista efectuada a una de las ingenieras que laboran en la oficina encargada de manejar las herramientas tecnológicas que se aplican a los cursos de ley en la Escuela.

Los resultados de la encuesta evidenciaron que el $58,5 \%$ de los estudiantes encuestados conocen las herramientas tecnológicas con las que cuentan en los cursos de ley. Así mismo, fue posible confirmar que la plataforma más conocida por los capitanes es la CEDOC 360 (26\%), seguida de la Blackboard (18\%), mientras que las menos reconocidas son las herramientas de Google (Google Forms, Google Académico, etc.).

Del mismo modo, el 61\% de los estudiantes saben cuáles son las herramientas tecnológicas aplicadas en su curso y las relacionan con los links de tareas, foros académicos y video conferencias. Esto es coherente con el hecho de que el 67\% afirma no haber presentado evaluaciones de tipo académico en línea y de que el 63,4\% manifiesta no haber recibido ningún tipo de capacitación para el manejo de los ambientes virtuales. Sin embargo, afirman que los instrumentos funcionan bien.

Por otro lado, el 51\% indica que las herramientas tecnológicas del curso son fáciles de entender y navegar. El 53\% dice que las herramientas son apropiadas, pero la mayoría de ellos sugieren que es necesario crear una aplicación para dispositivos móviles, realizar capacitaciones previas para su manejo y complementar las existentes con más funciones para los cursos.

La entrevista (anexo 2) a una de las ingenieras que forman parte de la oficina encargada de manejar las herramientas tecnológicas de la ESACE mostró que no se está evaluando a través de plataformas en línea, aunque sí existe la propuesta de utilizar alguna página académica para implementar este tipo de ayudas.

Asimismo, se evidenció que la ESACE cuenta actualmente con las dos herramientas tecnológicas señaladas anteriormente: la Blackboard y la CEDOC 360. La primera se utiliza como medio de consulta, ya que a través de ella los alumnos pueden acceder a bibliotecas y repositorios académicos para elaborar tareas y 
trabajos. Por su parte, la Blackboard es emplea para hacer encuestas y evaluaciones que solo son aplicables al personal en curso de Comando, quienes realizan cursos virtuales básicos y en ellos desarrollan evaluaciones virtuales con formatos de respuesta falso / verdadero, selección múltiple o apareamiento.

En lo que respecta a la CEDOC 360, se halló que esta plataforma es vista como un espacio para acceder al Classroom, al correo institucional, a bibliotecas y para ingresar por un link directo a las plataformas de Sofiaplus, que forman parte del convenio institucional que existe con el Servicio Nacional de Aprendizaje (SENA). Sin embargo, este enlace no está habilitado para todos los estudiantes porque solo pueden acceder a él quienes se encuentran inscritos en el curso de inglés. Asimismo, es importante mencionar que a través del Classroom se tiene acceso para subir las tareas asignadas por los docentes.

Para finalizar, de acuerdo con las afirmaciones de la ingeniera, la evaluación del conocimiento de los capitanes que forman parte del curso de ascenso en la ESACE aún se realiza de manera escrita o en fotocopias, es decir, no existe registro de evaluaciones escritas.

\section{Discusión}

\section{Encuesta}

La encuesta muestra la perspectiva de los estudiantes y lo que ellos opinan acerca de las herramientas tecnológicas implementadas en el curso intermedio (Blackboard y CEDOC 360). A partir de la información recogida con este instrumento se estableció que más de la mitad de ellos conocen las herramientas tecnológicas que se aplican en la Escuela. Del mismo modo, se encontró que las más conocidas por los estudiantes son la CEDOC 360, la Blackboard y el Classroom, además saben que estas herramientas son empleadas en sus cursos.
Hasta ahora, el contexto en el que se desarrolla la encuesta muestra que los estudiantes están informados de las herramientas web y que son conscientes de que las utilizan en su proceso de formación. No obstante, este conocimiento viene del uso que hacen a diario de ellas, lo cual se relaciona con que no mencionen otras herramientas web como videoconferencias, foros, chats o incluso aplicaciones celulares que también pueden ser utilizadas con fines académicos.

Esta información se confirma con el hecho de que la mayoría de estudiantes relacionen estas herramientas con los links para cargar tareas, pero solo el 12\% con exámenes en línea. Estos resultados y que la mayoría manifieste que no ha presentado evaluaciones en línea durante el curso evidencia que el proceso de evaluación académica necesita ser complementado con otro método de evaluación que está incluido en estas herramientas. Además, los estudiantes informan que no han recibido capacitación para manejar las plataformas; tal vez en el caso de la plataforma CEDOC 360 se espera que los estudiantes accedan por voluntad propia al módulo de guía de navegación de la plataforma sin percatarse de que esto causa molestias o complica el acceso o la información sobre ciertas funciones.

Finalmente, y, aunque la mayoría de ellos considera que las plataformas son fáciles de navegar, funcionan regularmente bien y que son buenas para sus procesos de aprendizaje, la mayoría sugiere que es necesario mejorar estas herramientas por medio de aplicativos celulares con los cuales puedan acceder a plataformas como la CEDOC 360 desde sus celulares. Asimismo, los estudiantes consideran que es importante recibir capacitaciones previas para comprender su manejo y, de esta manera, facilitar la comprensión y el manejo de las aulas virtuales. Cabe señalar que estos hallazgos constituyen parámetros para identificar cuál es la 
herramienta más apropiada para evaluar el desempeño académico de los alumnos de la ESACE.

La importancia de esta encuesta radica en que permite encontrar las fallas que puedan tener las herramientas tecnológicas que implementa la ESACE actualmente. Igualmente, proporciona la información necesaria para responder a la pregunta de investigación —¿¿cuál es la herramienta tecnológica apropiada para evaluar el desempeño académico de los capitanes en la ESACE?-, por cuanto evidencia las principales características que tiene este instrumento tecnológico para evaluar el desempeño académico en la totalidad de la oferta académica de la ESACE.

Finalmente, es importante mencionar que la información proporcionada por los estudiantes también sirve como punto de partida para proponer una innovación educativa, ya que dan cuenta de cuál de las herramientas que se implementan actualmente en la ESACE puede ser complementada con nuevas utilidades para que hacer la evaluación académica en la ESACE.

\section{Entrevista}

Igual que sucede con la encuesta, la entrevista al personal de la oficina encargada del manejo de los instrumentos tecnológicos en la ESACE proporcionó otro punto de vista sobre la Blackboard y la plataforma CEDOC 360. También permitió encontrar una respuesta a la pregunta de investigación gracias a la información que el personal facilitó respecto a los nuevos procesos y las proyecciones de la Escuela sobre estas herramientas. Claro está, los datos suministrados por esta oficina muchas veces son ignorados por los alumnos, lo cual contribuye para alinear las respuestas con las de los capitanes que respondieron la encuesta.

Con la información proporcionada por la ingeniera fue posible conocer las fallas que la oficina ha identificado, las proyecciones de la Escuela sobre la educación militar de los oficiales en curso de ascenso y la manera como está enfocando estas herramientas para llevar a cabo procesos de enseñanza más transparentes y continuos. Asimismo, es importante resaltar que la entrevista confirmó la inexistencia de una herramienta útil para realizar evaluaciones en línea, lo cual evidencia la problemática en la ESACE y, por lo tanto, la viabilidad del objetivo de investigación.

\section{Propuesta: Herramienta tecnológica para evaluar el desempeño académico aplicable en los cursos de ascenso en la ESACE}

\section{Descripción de la herramienta}

El Plan Estratégico del Sistema Educativo (Pese) (Ministerio de Defensa Nacional, 2008) promueve la certificación de la calidad de la educación militar y policial a partir de la vinculación de los procesos de enseñanza, aprendizaje y evaluación con la tecnología y otras herramientas que modernizan las instituciones. En este sentido, es evidente que la gestión educativa tiene el deber de incluir herramientas web en todos los procesos y subprocesos de la formación en la ESACE. En respuesta a esta necesidad, esta investigación propone que se utilice la plataforma CEDOC 360 mejorada.

A primera vista podría parecer que no se propone nada nuevo, sin embargo, los resultados de la revisión documental y los datos obtenidos de la entrevista y las encuesta demuestran que la plataforma CEDOC 360 cuenta con las bases necesarias para ser la herramienta adecuada de evaluación académica en la ESACE. No obstante, requiere ciertas modificaciones que van a mejorar su rendimiento y a ofrecer 
al estudiante nuevas funciones que facilitan los procesos y hará más transparente la evaluación de los conocimientos de los alumnos.

No obstante, surge la inquietud sobre cómo se puede mejorar esta herramienta para satisfacer las necesidades inmediatas de los alumnos y facilitar el trabajo de los docentes, quienes van a tener más herramientas para evaluar a sus estudiantes. La respuesta es el sistema Ultra ${ }^{1}$, que se encuentra en las plataformas Blackboard y con el cual se pueden crear cuestionarios en línea que no solo ponen a prueba los conocimientos de los estudiantes, sino que además dota al docente con una herramienta que permite sacar calificaciones inmediatas y medir el promedio del curso comparando resultados con otros ciclos u otras pruebas sin ningún tipo de influencia externa o manipulación. Estas características aseguran un proceso transparente para seguir los principios de la plataforma CEDOC 360.

Esta alternativa es viable por cuanto una de las características de la plataforma CEDOC 360 es que permite vincular las plataformas Blackboard en su contenido, lo cual facilitaría que se creen módulos de materias diferentes a la investigación y que se hagan evaluaciones del curso, e incluso de mayor envergadura como los Exámenes de Competencia con rapidez y trasparencia. Además, los costos en papelería disminuyen y se alinean con la política medioambiental de la Escuela y de las Fuerzas Armadas.

Es importante mencionar que, como complemento a la implementación de las evaluaciones en línea, se sugiere crear una aplicación celular de CEDOC 360 y realizar jornadas de capacitación para acceder y manejar esta herra-

1 Ultra es una herramienta vinculada a Blackboard con la que se pueden crear cuestionarios en línea y páginas de evaluación dentro del contenido de la plataforma (Blackboard, s. f.). mienta y la plataforma Blackboard, así como para desarrollar las evaluaciones en línea.

\section{Objetivo}

Proporcionar a los estudiantes una herramienta web que agilice y mejore los métodos de evaluación de conocimientos en los cursos de ascenso ofrecidos en la ESACE.

\section{Actores}

A continuación, se presentan los principales actores de esta propuesta:
a. Ministerio de Defensa Nacional
b. Comando General de las FF. MM.
c. Comando Ejército
d. Centro de Educación Militar
e. Dirección de la Escuela de Armas Combinadas del Ejército

\section{Proceso de implementación}

El proceso debe iniciar con la presentación de la propuesta, la obtención de recursos para crear nuevos módulos de Ultra en la plataforma CEDOC 360, la elaboración del material e inclusión en este espacio web. Sumado a esto, de forma paralela a la aplicación de Ultra se deben crear capacitaciones para el manejo de las nuevas funciones de la plataforma y el acceso a los exámenes. Posteriormente, se deberá hacer la propuesta de la aplicación celular para que los estudiantes puedan tener acceso desde sus dispositivos móviles (figura 4).

\section{Alcance y resultados esperados}

Con la implementación de estas mejoras y la puesta en marcha de los exámenes en línea se espera mejorar los procesos académicos de la ESACE, reducir el tiempo de respuesta en la calificación de exámenes y consolidar la transparencia en los procesos de calificación de los cursos de ley. Del mismo modo, con esta herramienta se espera vincular la educación ofrecida 


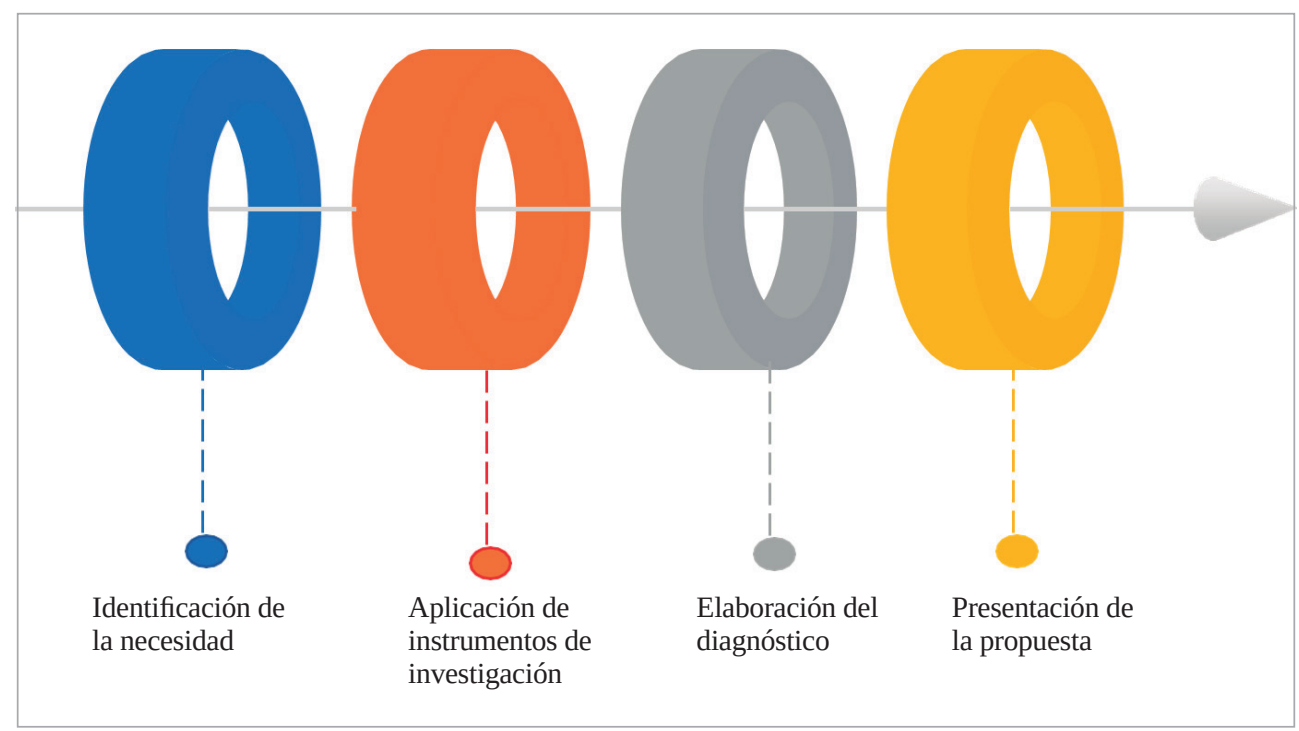

Figura 4. Proceso de implementación de la herramienta.

Fuente: Original de los autores.

en las escuelas de formación del Ejército al proceso de modernización y transformación institucional promovido en la Doctrina Damasco y formar profesionales capaces de responder a los retos que la nueva sociedad y el entorno cambiante de Colombia les presentan.

\section{Conclusión}

En primer lugar, la revisión de los procesos de evaluación de desempeño académico en la ESACE se fundamentó en la revisión de la normatividad y doctrina vigente definida por el Ministerio de Defensa, ente rector en el establecimiento de parámetros educativos en las Fuerzas Militares y de Policía. Esta revisión evidenció que la ESACE, como parte de las escuelas de formación del Ejército Nacional, debe promover la calidad educativa de la mano con la tecnología y modernizar los métodos evaluativos de acuerdo con las nuevas tecnologías. Así mismo, capacitar a los oficiales en escenarios que modernicen y diversifiquen sus herramien- tas para contribuir con el cumplimiento de la misión institucional desde su quehacer académico, de tal manera que se haga realidad el ideal educativo del "Héroe Bicentenario", capacitado para responder en el área de trabajo y en el espacio cibernético.

En segundo lugar, las herramientas implementadas en los cursos de ley que imparte la ESACE son una muestra del interés de los mandos por mejorar las condiciones educativas de los oficiales del Ejército Nacional. Es así como se han preocupado por crear una herramienta enfocada en el Ejército Nacional y en sus estudiantes que tiene el propósito de crear procesos transparentes y completos en los cuales los oficiales del Ejército desarrollen capacidades investigativas y contribuyan con la transformación y modernización institucional a través de la innovación y la investigación orientada a mejorar las acciones, condiciones de trabajo o estrategias aplicadas por los batallones y las unidades en el campo de trabajo. No obstante, esta 
investigación encontró que las dos plataformas (Blackboard y CEDOC 360) presentan falencias y generan dificultades para los estudiantes, quienes necesitan medios con más utilidades y que les proporcionen elementos para llevar a cabo un proceso inmediato de calificación y verificación de debilidades. Sin duda, recibir retroalimentación inmediata, el manejo de nuevas temáticas de manera virtual y la conexión de la labor militar y del liderazgo con la tecnología contribuye a que Colombia esté protegida por oficiales creativos, innovadores y propositivos que pueden actuar en muchos escenarios sin importar factores endógenos o exógenos que influyan en ellos.

La búsqueda de una herramienta tecnológica de evaluación apropiada para implementar en los cursos de ley incluidos dentro de la oferta académica de la ESACE se realizó con base en la opinión de dos actores directamente relacionados con el tema: los funcionarios a cargo de las plataformas virtuales y los alumnos que la utilizan. Así, conociendo el contexto real y sus opiniones, fue posible no solo encontrar los vacíos que tienen las herramientas actuales, sino además establecer que una de ellas presenta mayores ventajas para los alumnos: la plataforma CEDOC 360, impulsada por el Centro de Educación Militar, es la herramienta adecuada para desarrollar los procesos de aprendizaje de los oficiales en curso de ascenso. No obstante, aún presenta vacíos que se deben cubrir con otras herramientas, como la función Ultra de Blackboard, con la cual se abre la posibilidad de presentar evaluaciones en línea, obtener retroalimentación inmediata y conocer la calificación de forma rápida. De esta manera, se promovería la trasparencia en el proceso y se proporcionaría al docente y al estudiante instrumentos que faciliten su labor y la construcción de conocimiento a través de ayudas virtuales.

\section{Declaración de divulgación}

Los puntos de vista y los resultados de este artículo pertenecen a los autores y no reflejan necesariamente los de la Escuela Militar de Cadetes “General José María Córdova”.

\section{Financiamiento}

Los autores no declaran fuente de financiamiento para la realización de este artículo.

\section{Sobre los autores}

Jairo Augusto Vásquez Clavijo. Mayor del Ejército Nacional de Colombia. Especialista en Conducción y administración de unidades y Especialista en administración de recursos para la defensa nacional. Profesional en ciencias Militares. Contacto: jairo.velasquezcl@buzonejercito.mil.co

Braymer Sinay Velásquez Carrillo. Mayor del Ejército Nacional de Colombia. Especialista en conducción y administración de unidades y especialista en administración de recursos para la defensa nacional. Profesional en ciencias Militares.

Andrés David Villa Molano. Mayor del Ejército Nacional de Colombia. Especialista en conducción y administración de unidades y especialista en administración de recursos para la defensa nacional. Profesional en ciencias Militares y en Administración de Empresas.

Alexander Román Yela Garzón. Mayor del Ejército Nacional de Colombia. Especialista en conducción y administración de unidades y especialista en administración de recursos para la defensa nacional. Profesional en ciencias Militares. 


\section{Referencias}

Blackboard. (s. f.). Crear pruebas y encuestas. Recuperado de https://help.blackboard.com/es-es/ Learn/Instructor/Tests_Pools_Surveys/Create_ Tests_and_Surveys

Canales, R. (2007). Identificación de factores que contribuyen al desarrollo de actividades de enseñanza y aprendizaje con apoyo de las TIC, que resulten eficientes y eficaces. Análisis de su presencia en tres centros docentes (Tesis doctoral en Educación). Universitat Autònoma de Barcelona, Departament de Pedagogia Aplicada, Barcelona, España. Recuperado de https://www.tesisenred. net/handle/10803/5045\#page $=1$

Caro, L. (s. f). ¿Qué es el conocimiento tecnológico? Recuperado de https://www.lifeder.com/conocimiento-tecnologico/

Centro de Educación Militar (CEMIL). (diciembre 14 de 2017). CEDOC 360 para educación (video en línea). Recuperado de https://www.youtube.com/ watch?v=Kc7SfGhUPqo

Christen, M. \& Ortiz, S. (s. f). Herramientas TIC para la evaluación del aprendizaje, las competencias y las habilidades de estudiantes de bachillerato. Recuperado de http://www.prepa5.unam.mx/ wwwP5/encuentroEvaluacion/mesa1/2.HerramsTICparaEvaluacionAprendizaje.pdf

Dávila, H. (marzo de 2017). Estrategia pedagógica mediada por las TIC para mejorar el rendimiento académico en el área de matemáticas para estudiantes de grado octavo del Colegio Pablo Neruda (Trabajo de especialización). Universidad los Libertadores, Bogotá, D. C., Colombia. Recuperado de https://repository.libertadores. edu.co/bitstream/handle/11371/1471/davilahector2017.pdf?sequence $=1$ \&isAllowed $=\mathrm{y}$

Ejército Nacional de Colombia. (2017). MFE 1.0. El Ejército. Ejército Nacional de Colombia. Bogotá, D. C.: Centro de Doctrina del Ejército.

García, E. (1988). Una teoría práctica sobre la evaluación. Revista de Educación, (287), 233-253. Recuperado de https://dialnet.unirioja.es/servlet/ejemplar?codigo=1980\&info=open_link_ ejemplar

García, L. (s. f). Historia de la educación a distancia. Recuperado de https://www.biblioteca.org.ar/ libros/142131.pdf

García, R., De la O, T., Jiménez, Y. \& Angulo, J. (2015). Redefinición y configuración de la formación universitaria mediada por entornos virtuales de aprendizaje. En J. Angulo, J. Vales, C. Acosta \& R. García, Aportes y reflexiones sobre la educación mediada por tecnologías (págs. 145-161). México, D. F.: Instituto Tecnológico de Sonora. Recuperado de https://www.researchgate.net/ publication/292127688_Redefinicion_y_configuracion_de_la_formacion_universitaria_ mediada_por_entornos_virtuales_de_aprendizaje/link/56a8ff8c08ae2df82165005d/download

González, K., Tovilla, C., Juárez, I. \& López, M. (2017). Uso de tecnologías de la información en el rendimiento académico basados en una población mexicana de estudiantes de Medicina. Educación Médica Superior, 31(2). Recuperado de http://scielo.sld.cu/scielo.php?script=sci_arttext\&pid=S0864-21412017000200008

Guzmán, T. (2008). Las Tecnologías de la Información y la Comunicación en la Universidad Autónoma de Querétaro: propuesta estratégica para su integración (Tesis de doctorado en Educación). Universitat Rovira I Virgili, Tarragona, España. Recuperado de https://www.tesisenred.net/bitstream/handle/10803/8937/TESIS_TGF.pdf

Hernández Sampieri, R., Fernández Collado, C. \& Baptista Lucio, M. D. (2014). Metodología de la investigación (6. ${ }^{\mathrm{a}}$ ed.). México D. F.: McGrawHill Education.

Hernández, R. (2017). Impacto de las TIC en la educación: retos y perspectivas. Propósitos y Representaciones, 5(1), 325-347. doi:http://dx.doi. org/10.20511/pyr2017.v5n1.149

Hernández, S. (2008). El modelo constructivista con las nuevas tecnologías: aplicado en el proceso de aprendizaje. Universities and Knowledge Society Journal, 5(2), 26-35. Recuperado de https:// www.redalyc.org/pdf/780/78011201008.pdf

Lafuente, M. (2003). Evaluación de los aprendizajes mediante herramientas TIC. Barcelona, España: Universitat de Barcelona.

Londoño, S. (2015). Implementación de las Tecnologías de la Información y Comunicación en el personal de oficiales del Ejército Nacional (Trabajo de diplomado). Universidad Militar Nueva Granada, Facultad de Estudios a Distancia, Bogotá, D. C., Colombia. Recuperado de https://repository.unimilitar.edu.co/bitstream/handle/10654/6319/Implementacio\%c c\%81n\%20TICS\%20oficiales\%20Ejercito\%20. pdf?sequence=1\&isAllowed=y 
López, V. (2009). Evaluación formativa y compartida en Educación Superior: propuestas, técnicas, instrumentos y experiencias. España: Narcea.

Ministerio de Defensa Nacional. (2008). Plan Estratégico del Sistema Educativo en las Fuerzas Armadas 2007-2019. Recuperado de https://www. mindefensa.gov.co/irj/go/km/docs/Mindefensa/ Documentos/descargas/estrategia_planeacion/ desa_capital/Pagina/PESE_FINAL.pdf

Nieto, R. (2012). Educación virtual o virtualidad en la educación. Revista Historia de la Educación Latinoamericana, 14(19), 137150. Recuperado de https://www.redalyc.org/ pdf/869/86926976007.pdf

Organización de las Naciones Unidas para la Educación, la Ciencia y la Cultura [Unesco]. (2013). Enfoques estratégicos sobre las TICS en educación en América Latina y el Caribe. Santiago de Chile: Unesco. Recuperado de http://www. unesco.org/new/fileadmin/MULTIMEDIA/ FIELD/Santiago/images/tics esp.pdf

Organización de las Naciones Unidas para la Educación, la Ciencia y la Cultura (Unesco). (2017). Las Tecnologías de la Información y la Comunicación (TIC) en la Educación. Recuperado de http://www.unesco.org/new/es/havana/areas-ofaction/education/tic-en-la-educacion/

República de Colombia. (diciembre 28 de 1992). Ley 30 de 1992. Congreso de la República. Diario Oficial, 40.700. Recuperado de https:// www.mineducacion.gov.co/1621/articles-85860_ archivo_pdf.pdf

República de Colombia. (julio 30 de 2009). Ley 1341 de 2009. Congreso de la República. Diario Oficial, 47.426. Recuperado de https://mintic.gov. co/portal/604/articles-8580_PDF_Ley_1341.pdf

República de Colombia. (1991). Constitución Política de Colombia. Congreso de la República. Recuperado de http://www.constitucioncolombia.com
República de Colombia. (febrero 8 de 1994). Ley 115 de 1994. Congreso de la República. Diario Oficial, 41.214 Recuperado de https://www. mineducacion.gov.co/1621/articles- 85906_ archivo_pdf.pdf

Ríos, S. \& Krenn, E. (2015). La evaluación mediada por TIC: abordaje desde una perspectiva comunicacional. Ponencia presentada en el XVII Congreso de la Red de Carreras de Comunicación Social y Periodismo de Argentina. Córdoba, Argentina.

Rodríguez, M. (2005). Aplicación de las TIC a la evaluación de alumnos universitarios. Teoría de la Educación. Educación y Cultura en la Sociedad de la Información, 6(2), s. p.

Rojas, P. \& Sepúlveda, S. (1999). ¿Qué es la competitividad? Recuperado de http://unpan1. un.org/intradoc/groups/public/documents/icap/ unpan038655.pdf

Santos, M. (1999). Evaluación educativa. Un proceso de diálogo, comprensión y mejora (1. ${ }^{\text {a ed.). Río }}$ de La Plata: Editorial Magisterio.

Schorborgh. (07 de junio de 2009). Herramientas tecnológicas. Recuperado de https:// es.slideshare.net/schorborgh/herramientas-tecnolgicas-1543885

Toro, G. (noviembre de 2016). Enseñanza en educación superior: una aproximación a la evolución de la innovación en la enseñanza de disciplinas científicas, con énfasis en el uso de tic en ambientes de aprendizaje. Recuperado de https://www. tesisenred.net/handle/10803/400153\#page=1

Vence, L. M. (s. f.). Uso pedagógico de las TIC para el fortalecimiento de estrategias didácticas del programa “Todos a Aprender”. Atlántico, Colombia.

Vicerrectoría de Asuntos Académicos Universidad del Turabo. (s. f.). Guía de Blackboard para estudiantes. Recuperado de https://ut.suagm.edu/ sites/default/files/uploads/Educacion-distancia/ Estudiantes/guia-blackboard-del-estudiante.pdf 


\section{Anexo 1}

Ficha técnica de la encuesta

Persona natural o jurídica que la realizó: Ct. Jairo Augusto Vásquez Clavijo, Ct. Braymer Sinay Velásquez Carrillo, Ct. Andrés David Villa Molano y Ct. Alexander Román Yela Garzón, alumnos del curso de ascenso de capitán a mayor de la Escuela de Armas Combinadas (ESACE) del Ejército Nacional.

Temas a los que se refiere: reconocimiento e identificación de herramientas tecnológicas aplicables en la ESACE para verificar el nivel académico y los conocimientos obtenidos por los alumnos de los cursos de ley.

Universo poblacional: estudiantes del curso de ascenso en la ESACE.

Grupo objetivo: capitanes en curso de ascenso de capitán a mayor que estudian en la ESACE.

Sistema de muestreo: se implementó un muestreo aleatorio de conglomerados en el que se elige un universo, que en este caso es el grupo de estudiantes en curso de ascenso en la ESACE. Con base en esta muestra se dividió la población en varios conglomerados, que en este caso son cada uno de los cursos de capitanes que hacen el curso de ascenso a mayor. Posteriormente, se eligieron algunos de estos cursos para aplicar la encuesta y poder estudiarlos.

Marco muestral: la selección de la muestra inicia con el marco de muestreo, que incluye el conglomerado de estudiantes de la ESACE. Luego, el marco de muestreo se redujo a la lista de cursos de ascenso en la ESACE y posterior- mente se obtuvo el listado de grupos de capitanes en curso de ascenso a mayor, quienes cuentan con las mismas características y cumplen con el perfil necesario para llevar a cabo la encuesta y la exploración del tema.

Tamaño de la muestra: 146 encuestas distribuidas entre 234 capitanes que se encuentran haciendo curso de ascenso al siguiente grado inmediato.

Margen de error observado: el nivel de confianza de la investigación es del 95\% y el margen de error es del 5\%.

Ponderación: muestra ponderada de acuerdo con el grado y curso en el que se encuentra enlistado.

Técnica de recolección de datos: encuestas virtuales con cuestionario estructurado.

Fecha de realización: 4 al 20 de octubre de 2019

\section{Número de encuestadores: 4}

\section{Anexo 2}

\section{Ficha Técnica entrevista}

Dirección: la entrevista fue realizada por el capitán Jairo Augusto Vásquez Clavijo, estudiante del curso de ascenso de capitán a mayor del Ejército Nacional.

Técnica: el tipo de entrevista responde a la entrevista semiestructurada, en la cual se lleva un formato preestablecido que puede variar dependiendo de las respuestas del entrevistado.

Fecha de realización: miércoles 23 de octubre de 2019.

Entrevistada: ingeniera a cargo del manejo de plataformas virtuales de la ESACE. 